\title{
Turbulent Combustion Modeling in Supersonic Flows for future RBCC vehicles
}

\author{
By Antonella INGENITO and Claudio BRUNO \\ Department of Mechanics and Aeronautics, "La Sapienza”, University of Rome, Italy
}

(Received May 2nd, 2008)

\begin{abstract}
Mixing and combustion of supersonic reacting flows are currently under investigation for new generation RBCC vehicles. Because of the speed within the SCRJ combustor, the length required for fuel and air to mix and react is commonly thought to be a difficult compromise if friction drag is to be kept to a minimum. LES simulations can be an useful tool to better understand the physics of supersonic combustion. By simulating combustion and analyzing numerical results, it is possible to draw fundamental conclusions suggesting how to design a more efficient combustor. Thus the subgrid scale (SGS) model to be implemented as closure of the filtered Navier Stokes equations is critical in reproducing experimental results and giving credibility to numerical predictions. In the present paper, a new SGS model for supersonic reacting flows is being proposed. This model accounts for compressibility effects on mixing and combustion by introducing a complete equations for the subgrid kinetic energy transport. This equation accounts for the effect of heat release due to combustion, dilatation due to the compressibility of the fluid, and the pressure gradient that are responsible for the baroclinic term and then for streamwise vorticity and dissipation. The reaction rate accounts for the effect of the Mach number by means of the SGS Mach number squared. The effect of the small scales scalars fluctuations on turbulent mass diffusivity is included by modeling the local Schmidt number.
\end{abstract}

Key Words: Supersonic combustion, Scramjet, Compressible Mixing, LES subgrid scale model

\section{Introduction}

The effect of the Schmidt number in simulating reacting fluid dynamics must be understood to better reproduce experimental results. The assumption of a constant Schmidt number, implies that turbulent species diffusivity depends only on the turbulent velocity fluctuations. This assumption is justified when species fluctuations at fine scales are negligible. In reacting flows, this assumption is no longer justified. In fact, combustion occurs in fine turbulent structures, where fuel and oxidizer begin to mix at the near molecular level: thus fluctuations of species concentrations due to combustion cannot be neglected.

Turbulent diffusivity must take into account this effect. This means that the assumption of a constant turbulent Schmidt number $\left(\mathrm{Sc}_{\mathrm{t}}\right)$ does not reproduce well turbulent mixing in reacting flows and can induce widely different predictions of the flow dynamics.

Previous simulations by these authors ${ }^{1)}$, performed assuming a constant $\mathrm{Sc}_{\mathrm{t}}$, showed that this parameter has a strong impact on flame behaviour. In particular, simulations with $\mathrm{Sc}_{\mathrm{t}}=0.4$ and $\mathrm{Sc}_{\mathrm{t}}=0.6$ predicted a (mean) steady flame, whilst simulations with $\mathrm{Sc}_{\mathrm{t}}=0.7$ predicted a flame that oscillates periodically. Furthermore, simulations have shown that decreasing $\mathrm{Sc}_{\mathrm{t}}$ the flame is less stratified and no longer confined to the fuel/oxidizer interface.

These results pointed out that a subgrid scale model for the turbulent mass diffusivity is a key requirement to better reproduce experimental results ant to understand flame behaviour.

\section{SGS model for the turbulent Schmidt number}

The understanding of the small scales influence on the passive scalars transport is of great interest in LES, particularly whenever mixing is controlling. In the current SGS models, the turbulent Schmidt number is generally assumed constant over the entire fluid dynamic field. With this assumption, scalar fluctuations are supposed to be proportional to the local velocity fluctuations, and this proportionality is generally assumed to be constant everywhere.

In particular, in the case of species transport, the turbulent mass diffusivity is expressed as a function of the eddy viscosity $\mu_{t}$ and thus a function of the SGS velocity fluctuations divided by a constant parameter, the turbulent Schmidt number $\mathrm{Sc}_{t}$, that is

$$
D_{t}=\frac{v_{t}}{S c_{t}}
$$

where in the ISCM model ${ }^{4}$ used here the turbulent kinematic viscosity $v_{\tau}$ is $v_{t}=\sqrt{k_{s g s}} \Delta$.

This assumption implies that the scalar fluctuations are proportional to the local velocity fluctuations and this proportionality is constant everywhere. In fact, velocity fluctuations occurring near the dissipative scale (fine 
structures) enhance species transport, and at large scales the effect is experienced as a greater mass diffusivity.

Actually, the turbulent mass diffusivity is affected not only by the convective transport due to microscale velocity fluctuations, but also by local scalar fluctuations. In fact, combustion within fine structures drive rapid changes of local species concentrations. This effect cannot be neglected when modelling species transport due to local turbulence.

Where combustion does not occur, it is still possible to assume that the turbulent species transport is proportional to the velocity fluctuations and the main contribution to the turbulent diffusivity is then given by small scale velocity fluctuations. Where combustion does occur, not only the contribution of the subgrid kinetic energy to the species transport must be taken into account, but also that due to the species fluctuations within the fine structures.

Thus, due to the reactive fine structures, the turbulent mass transport within each computational cell is due at least to two contributes:

1. that given by velocity fluctuations due to combustion, dissipation and dilatational terms;

2. that given directly by species fluctuations driven by local combustion.

In fact, because of velocity fluctuations at the sub-grid scale, species are convected from one side to the other of the flame region. When combustion occurs, inside fine structures some species are produced and others are destroyed: the gradients so created contribute to the turbulent species transport. This contribution must also be modeled and implemented in the SGS model.

The species fluctuations due to the fine structures can be modeled considering that, because of the fine structures, the mixture mean weight within the computational cell is different than that calculated by the filtered Navier Stokes equations. In particular, assuming $\gamma^{*}$ as the volume fraction occupied by the fine structures within the entire cell volume, the correct value of the mixture mean weight, $W_{n}$, is:

$$
W_{n}=\gamma^{*} W_{s g s}+\left(1-\gamma^{*}\right) W_{c e l l}
$$

where $W_{s g s}$ is the mixture mean weight of the fine structures and $W_{\text {cell }}$ is the mixture mean weight in the computational cell, calculated directly by the filtered Navier Stokes equations.

The contribution due to the species fluctuations can be estimated as the difference between the 'correct' cell weight and that calculated using the filtered Navier Stokes equations:

$$
W_{n}-W_{\text {cell }}=\gamma^{*}\left(W_{\text {sgs }}-W_{\text {cell }}\right)
$$

These fluctuations contribute to the turbulent species diffusivity, and their contribute can therefore be modeled by:

$$
D_{t}=\sqrt{k_{s g s}} \Delta\left[1+\gamma^{*}\left(W_{s g s}-W_{\text {cell }}\right)\right]
$$

In Eq. (4) the turbulent diffusivity is no longer proportional only to the SGS turbulent viscosity: the contribution of species fluctuations is also accounted for. Depending on these local fluctuations the turbulent Schmidt number varies, its expression being:

$$
S c_{t}=\frac{1}{\left[1+\gamma^{*}\left(W_{\text {sgs }}-W_{\text {cell }}\right)\right]}
$$

\section{Large Eddy Simulations of Supersonic Combustion}

\subsection{The NASA-Langley Test Case: Geometry and Experimental Results}

The geometry of the SCRJ combustor configuration is shown in Fig. 1. A direct-connect supersonic combustor, in the testing campaign known by its acronym SCHOLAR, was built and instrumented at NASA-Langley Research Center. This configuration is that used during the NATO ATV-10 RTO $^{2)}$ project a few years ago.

This experiment was designed to provide optical access to a reacting supersonic flowfield typical of the flow present in a notional scramjet engine.
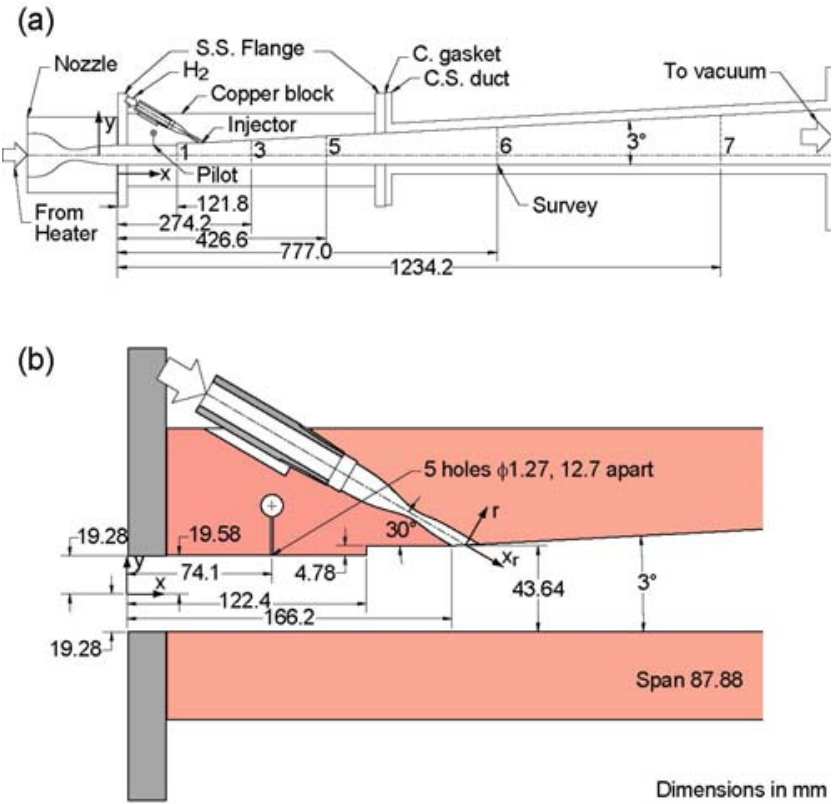

Fig. 1: Test model:

(a) nozzle and combustion chamber,

(b) detail in the vicinity of the fuel injector

The model shown in Fig. 1 consists of a section 546 $\mathrm{mm}$ in length, constructed of copper (for thermal control), followed by a $914 \mathrm{~mm}$ long carbon steel section attached to the aft end of the copper section. The copper section contains a single fuel injector that introduces gaseous 
hydrogen into the vitiated air stream flowing through the model. The injector region of the combustor model is shown in Fig. 1.b. The model consists of a constant area channel $38.6 \mathrm{~mm}$ high and $87.9 \mathrm{~mm}$ wide followed by a $4.8 \mathrm{~mm}$ rearward-facing step and a $43.8 \mathrm{~mm}$ long constant area section. Combustion-heated vitiated air is introduced into the channel at Mach 2, $1184 \mathrm{~K}$, and $100405 \mathrm{~Pa}$.

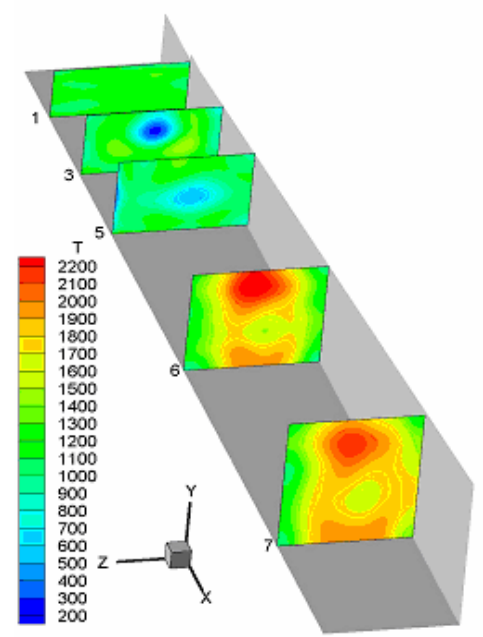

Fig. 2 3D view of the experimental average temperature field

The vitiated air contains 20.35 percent (mass) water introduced by the facility heater. The injector lies on the duct centerline. The fuel injector is inclined 30 degrees with respect to the horizontal and has a circular cross-section $7.6 \mathrm{~mm}$ in diameter. It is located just downstream of the $3^{\circ}$ diverging ( $43.8 \mathrm{~mm}$ from the step) section. The $3^{\circ}$ expansion of the upper wall begins immediately at the primary fuel injector and continues along the upper wall of the carbon steel section, see Fig. 1 a.

Five measurement locations for optical access to CARS diagnostics are provided in the copper section of the combustor. Two additional measurement stations are provided in the carbon steel section. From these single-shot measurements, averages and RMS values are derived. In additional to optical measurements, wall pressures are measured using an array of orifices. In Fig. 2 a $3 \mathrm{D}$ view of the experimental average temperature field is shown. Experiments predict flame anchoring between cross sections 5 and 6 . As this last section is at $77 \mathrm{~cm}$ from the combustor inlet plane, and at about $60 \mathrm{~cm}$ from the $\mathrm{H}_{2}$ injector, mixing times can be estimated to be $5 \times 10^{-4} \mathrm{~s}$ : this means that the supersonic flow investigated (see inlet parameters below) mixes and burns in a very reasonably short time. Fig. 2 shows that the flame develops both at the bottom and upper wall. Maximum (averaged) temperature measured are 2200 to $2300 \mathrm{~K}$.

\subsection{Numerical Scheme}

Three-dimensional Large Eddy Simulations were performed with the FLUENT $6.3{ }^{\mathrm{TM}}$ commercial code ${ }^{3)}$ to simulate the flowfield. Double precision has been used.

The LES solver is a coupled, upwind, explicit, third-order MUSCL, accurate in space. This scheme was conceived from the original by blending a central difference scheme and second-order upwind scheme. Compared to the second-order upwind scheme, the third-order MUSCL has the potential to improve spatial accuracy for all types of mesh by reducing numerical diffusion, most significantly for complex three-dimensional flows.

\subsection{Computational Domain}

The SCRJ combustor geometry of Fig. 2 has been mapped by a 3-D grid.

This domain was discretized with a grid consisting of 700 points in the streamwise direction, 46 points in the cross-stream direction, and 60 points in the spanwise direction. The grid was made finer near the step and the fuel injector. The number of hexahedral cells of the computational domain is 1,563,994, for a total of $1,626,578$ nodes. Only the first $800 \mathrm{~mm}$ of the whole length of the combustor (1234.2 $\mathrm{mm}$ ) have been simulated. With this grid, a very small wall $\mathrm{Y}^{+}$, of order of 4 - 8 has been obtained near the step and the $\mathrm{H} 2$ injector, where the NASA experiment predicts bow shock formation. At the inlet instead, the grid should be further refined to allow a better prediction of the boundary layer. Generally speaking, this grid is not accurate enough to capture all fluid dynamics details (e.g., BL transition, or the fine details of SBLI); however, emphasis here is on simulating flame development and anchoring by means of the specially-developed SGS mentioned above. Furthermore, results in [6] for the same combustor and with a similar grid indicate the grid size used is adequate to predict combustor performance. In fact, both the shock departing from the step and that just upstream the fuel injection are well predicted by the simulations performed, leading to the formation of two counter-rotating vortices moving with the stream direction. Shocks reflection between upper and bottom walls is also well predicted.

\subsection{Inlet Boundary Conditions}

Numerical simulations with the ISCM model have included only $800 \mathrm{~mm}$ of the whole length of the SCHOLAR combustor $(1200 \mathrm{~mm})$ : the inlet plane $\mathrm{X}=0$ in the simulations corresponds to the plane $\mathrm{X}=77 \mathrm{~mm}$ of the experiments.

Calculations begin at the $\mathrm{x}=0$ station of the SCHOLAR model, where vitiated air from the facility enters the duct. The initial channel cross-section is $38.6 \mathrm{~mm}$ high and 87.9 mm wide. Vitiated air entered the model at Mach 2.0 (velocity $1395.7 \mathrm{~m} / \mathrm{s}$ ), static temperature $1204 \mathrm{~K}$, and static pressure $101325 \mathrm{~Pa}$. Air inlet temperature is sufficiently high to produce self-ignition of the air-hydrogen mixture.

The mole fractions of the species present in the vitiated air are given in Table 1 : 
Table 1 Vitiated air mole fractions at the inlet

\begin{tabular}{|cl|c|}
\hline $\mathrm{H}_{2}$ & mole fraction & $1.3 \times 10^{-5}$ \\
\hline $\mathrm{O}_{\mathrm{H}}$ & mole fraction & 0.00071 \\
\hline $\mathrm{O}_{2}$ & mole fraction & 0.21 \\
\hline $\mathrm{H}_{2} \mathrm{O}$ & mole fraction & 0.217 \\
\hline $\mathrm{N}_{2}$ & mole fractions & 0.5722 \\
\hline
\end{tabular}

The hydrogen fuel injector introduces hydrogen at Mach 2.5, at static temperature $1343 \mathrm{~K}$, and at static pressure $202650 \mathrm{~Pa}$. Air $/ \mathrm{H}_{2}$ equivalence ratio is $\sim 1$.

Combustor walls are non-adiabatic; in fact, the measured heat flux varies linearly from $0.7 \mathrm{MW} / \mathrm{m}^{2}$ to $1.8 \mathrm{MW} / \mathrm{m}^{2}$ along the $\mathrm{x}$ direction, corresponding to a loss of about $10 \%$ of the combustion heat release. The heat flux function has been implemented as BC for the energy equation by means of a user defined function in the code used for these simulations.

No-slip conditions were specified along the channel walls.

\subsection{Turbulence and chemistry modelling}

The subgrid scale model used for the turbulent closure of the filtered Navier Stokes equations includes turbulent viscosity and turbulent diffusivity calculated by the ISCM model $^{4}$ and introduced in the FLUENT 6.3 code by means of a user defined function. In brief, in the ISCM model:

1. micro-scale physics has been included by means of a subgrid kinetic energy equation that is algebraically modelled to provide the velocity fluctuation to be used for an eddy viscosity SGS closure;

2. reacting turbulent structures have been modelled by means of a reactor burning at locally constant volume;

3.the reaction rate depends on the local Mach number ${ }^{4)}$

4. the turbulent diffusivity is a function of the velocity and species fluctuations, and is calculated as described in Section II.

5. The Prandtl turbulent number has been assumed constant and equal to 0.8 .

6. Hydrogen/air chemical kinetics has been modelled by means of a global 1-step reaction and 3 reacting species ${ }^{5}$ :

$$
\begin{gathered}
\mathrm{H}_{2}+1 / 2 \mathrm{O}_{2}=\mathrm{H}_{2} \mathrm{O} \quad \mathrm{k}_{\text {global }}=1.8 \times 10^{13} \\
\exp (-17614 / \mathrm{T})\left[\mathrm{H}_{2}\right]^{1.0}\left[\mathrm{O}_{2}\right]^{0.5}
\end{gathered}
$$

This kinetic scheme for $\mathrm{H}_{2}$ /air combustion does not account for the effects of the small quantities of radicals species present in the vitiated air. That, and the assumption of 1-step mechanism instead of a more detailed kinetics is expedient to save computational time, but can be justified noting that flame anchoring at the air temperature and pressure of these simulations (and the measurements) is not controlled by kinetics but by mixing.

In fact, results obtained by detailed hydrogen/air kinetics simulations (with 9 species and 20 reactions) using the CHEMKIN 3.7 code and for the same NASA-Langley test case conditions, show ignition delay times of order $0.05 \mathrm{~ms}$, ten times lower than the mixing time extracted from the experiments. This result is true for the SCHOLAR experiments simulated here; in general, for lower air inlet temperature or pressure, full $\mathrm{H}_{2}$ /air kinetics should be introduced in simulations.

\subsection{Simulations with the ISCM model and variable $\mathrm{Sc}_{\mathbf{t}}$}

Numerical simulations with the new variable turbulent Schmidt number show results in better agreement with SCHOLAR data. As found also in 6), the computed results are highly dependent on the compressibility correction for the turbulence model. When the effect of compressibility on mixing and on reaction rate are neglected, f.i., by implementing the Smagorinky-Lilly SGS model ${ }^{7)}$, combustion does not occur.

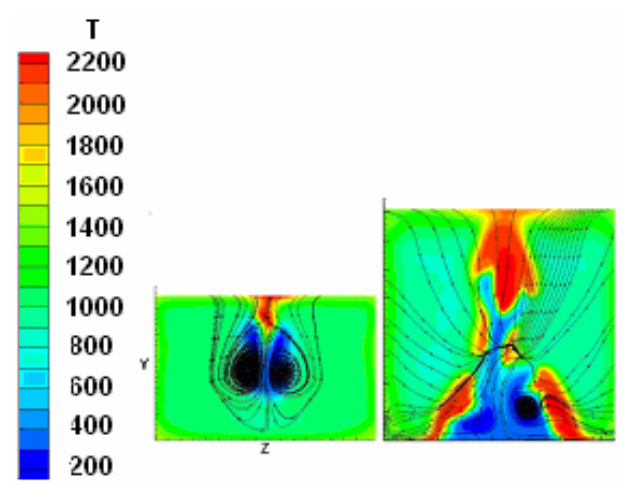

Fig. 3 Instantaneous temperature field in the $\mathrm{YZ}$ plane at $\mathrm{X}=0.35 \mathrm{~cm}$ and $\mathrm{X}=0.77$

\section{$\mathbf{T}$}

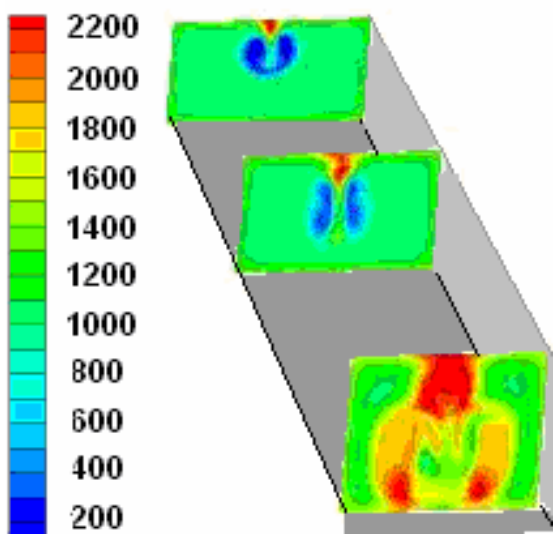

Fig. 4 Averaged temperature field in the $\mathrm{YZ}$ plane at $\mathrm{X}=0.2 \mathrm{~cm}$ and $\mathrm{X}=0.35 \mathrm{~cm}, \mathrm{X}=0.77 \mathrm{~cm}$

Fig. 3 and Fig. 4 show averaged and instantaneous temperature fields in sections 5, 6 and at the exit. A more distributed combustion (with respect to simulations 
with uniform $\mathrm{Sc}_{\mathrm{t}}=0.7$ ) is predicted. As shown by the experimental results, the flame is no longer confined only near the $\mathrm{H}_{2}$ /fuel interface,. In these figures, two counter-rotating vortices are seen moving along the stream direction and straining the $\mathrm{H}_{2}$ jet.

Averaged and instantaneous temperature fields in the middle XY plane are shown in Fig. 5 and Fig. 6 .

These figures shows that vorticity is mainly streamwise; the flame reaches the end of combustor and continues out. The maximum instantaneous temperature is about $2610 \mathrm{~K}$, while the maximum averaged temperature predicted with variable $\mathrm{Sc}_{\mathrm{t}}$ number is close to $2350 \mathrm{~K}$, and in any case no higher than $2560 \mathrm{~K}$. Temperature fluctuations are of order $5 \%$. In 1 ), fluctuations of temperature are of order $10 \%$.

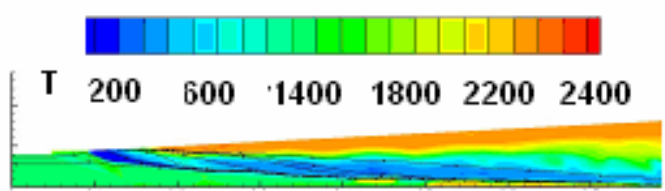

Fig. 5 Averaged temperature field in the middle XY plane

The results also indicate that the flame field is of course unsteady, but not subject to periodic large-amplitude oscillations: once ignited the flame does not quench periodically, as when assuming a constant $\mathrm{Sc}_{\mathrm{t}}=0.7^{1)}$.

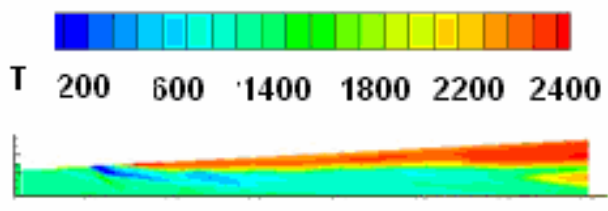

Fig. 6 Instantaneous temperature field in the middle XY plane

Fig. 7 shows the turbulent Schmidt number predicted by the ISCM model. It varies from 0.01 to 0.45 , but $\mathrm{Sc}_{\mathrm{t}}=0.25-0.35$ prevail in most of the field. Note these numerical values are the effect of the particular way $\mathrm{Sc}_{\mathrm{t}}$ has been defined in Section 2 .
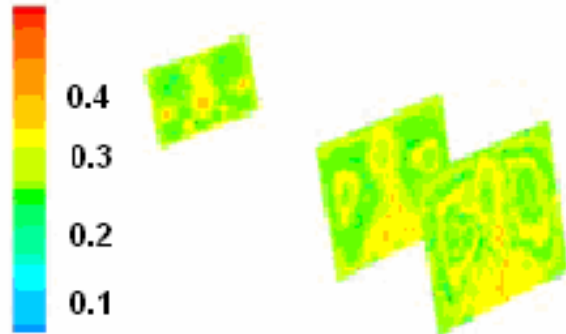

Fig. 7 Instantaneous $\mathrm{Sc}_{\mathrm{t}}$ field in the $\mathrm{YZ}$ plane at $\mathrm{X}=0.3 \mathrm{~cm}, \mathrm{X}=0.7 \mathrm{~cm}$ and $\mathrm{X}=0.8 \mathrm{~cm}$
The turbulent $\mathrm{Sc}_{\mathrm{t}}$ predicted in the field is lower than the conventional 0.7 value typically assumed in the whole field, and justifies the stabler flame with respect to results obtained with constant $\mathrm{Sc}_{\mathrm{t}}=0.7$ : lower $\mathrm{Sc}_{\mathrm{t}}$ increase turbulent transport and promote mixing.

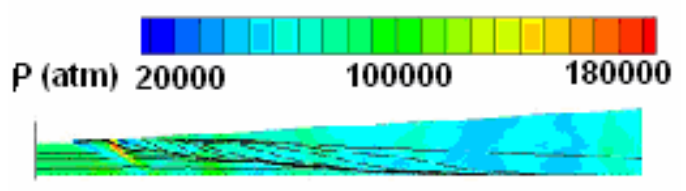

Fig. 8 Instantaneous pressure field in the median XY plane

Fig. 8 shows qualitatively the complex shock-boundary layer interaction and shock reflection that extends upstream of the backward facing step (details are in Fig. 9).

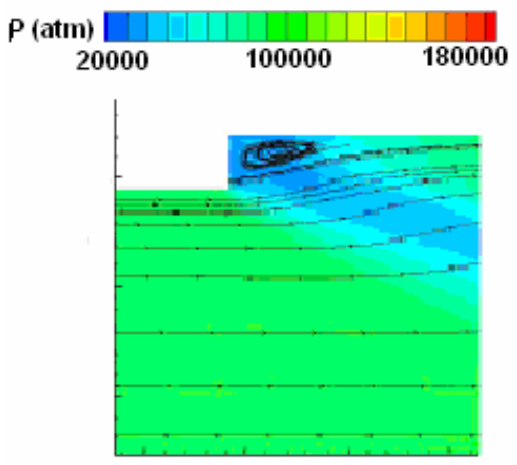

Fig. 9 Zoom of the instantaneous pressure field near the step

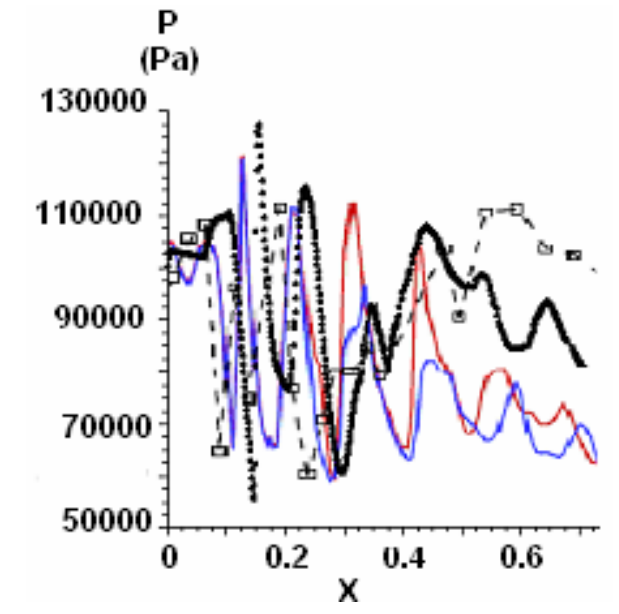

Fig. 10 Comparison of the pressure distribution along centerline of bottom wall obtained with the ISCM model (black line), Connaire kinetic sheme results by Keistler (red), Jachimowski kinetic sheme results by Keistler (blue) and the experimental results (broken line).

As for the pressure distribution along the centerline of the bottom wall, Fig. 10 shows experimental pressure 
maxima of $110,000 \mathrm{~Pa}$ at $\mathrm{X}=0.15, \mathrm{X}=0.2, \mathrm{X}=0.25$ and $\mathrm{X}=0.67$, and a minimum pressure $70,000 \mathrm{~Pa}$ at the exit, due to the expansion of the combustor cross section area.

The first three pressure peaks are located at the same locations of the experiments, but the second peak is overpredicted. Numerical results predict the last pressure peak is underpredicted: $85,000 \mathrm{~Pa}$ at $\mathrm{X}=0.45$, while experiments show $100,000 \mathrm{~Pa}$ at $\mathrm{X}=0.67$. Simulations in 6) using Jachimowski's H2/air scheme (7 species and 7 reactions) and the Connaire scheme (9 species and 19 reactions) predict at the same location $65000 \mathrm{~Pa}$ in both cases: aside from the numerical differences, that confirms that chemical kinetics is not crucial in this test case, and that combustion is indeed mixing-controlled.

\subsection{Conclusions}

In this work LES of supersonic combustion with a SGS model that accounts for the effect of compressibility on combustion and mixing has been performed. A variable turbulent Schmidt number model based on fundamental physics has been implemented in the calculations. The range of the $\mathrm{Sc}_{\mathrm{t}}$ predicted by this model at different points of the computational field is from 0.1 to 4 . The variable $\mathrm{Sc}_{\mathrm{t}}$ model shows improved results over the constant $\mathrm{Sc}_{\mathrm{t}}$ assumption: in particular, the flame is more distributed, in agreement with experimental results. Numerical results predict an unsteady but not unstable flame, with averaged temperatures close to those measured in the experiments. Similarly, he pressure distribution on the bottom wall is very well matched. Future work will include detailed kinetics implementation and improving the SGS model for energy transport.

\section{Acknowledgments}

This work was performed within the 'Long-Term Advanced Propulsion Concepts and Technologies' (LAPCAT) project investigating high-speed airbreathing propulsion. LAPCAT, coordinated by ESA-ESTEC, is supported by the EU within the 6th Framework Programme Priority 1.4, Aeronautics and Space, Contract no. AST4-CT-2005-012282. Further info on LAPCAT can be found on http://www.estec.esa.int/techresources/lapcat.

\section{References}

1) Ingenito, A. and Bruno, C.: Effect of the Turbulent Schmidt Number on Supersonic Regime, 46th AIAA Aerospace Sciences Meeting and Exhibit, Reno, NV, 2008.

2) Drummond, J. P., Diskin, G. S. and Cutler, A. D.: Fuel-Air Mixing and Combustion in Scramjets, Technologies for Propelled Hypersonic Flight, NATO Research and Technology Organization Final Report, 2001.

3) http://www.fluent.com.

4) Ingenito, A., De Flora, M. G., Giacomazzi, E., Bruno, C. and Steelant, J.: A Novel Model of Turbulent Supersonic Combustion: Development and Validation, 42nd AIAA/ASME/SAE/ASEE Joint Propulsion Conference and
Exhibit, Sacramento, California, 2006.

5) Marinov, N. M., Westbrook, C. K. and Pitz, W. J.: Detailed and Global Chemical Kinetics Model for Hydrogen, Transport Phenomena in Combustion, Taylor and Francis, Washington, DC, 1 (1996), pp. 118-129.

6) Keistler, P. G.: Simulation of Supersonic Combustion Using Variable Turbulent Prandtl/Schmidt Numbers Formulation, PhD Dissertation, http://www.lib.ncsu.edu/theses/ available letd-02162007-101728/unrestricted/etd.pdf, 2001.

7) Ingenito, A., De Flora, G., Giacomazzi, E., Bruno, C. and Steelant, J.: LES Modeling of Scramjet Combustion, 44th AIAA Aerospace Sciences Meeting and Exhibit, Reno, NV, 2006. 